\title{
Magnetic confinement of the superconducting condensate in superconductor-ferromagnet hybrid composites
}

\author{
W. Gillijns, ${ }^{1, *}$ A. Yu. Aladyshkin, ${ }^{1,2}$ A. V. Silhanek, ${ }^{1}$ and V. V. Moshchalkov ${ }^{1}$ \\ ${ }^{1}$ INPAC, Institute for Nanoscale Physics and Chemistry, Nanoscale Superconductivity and Magnetism and Pulsed Fields Group, \\ K. U. Leuven, Celestijnenlaan 200D, B-3001 Leuven, Belgium \\ ${ }^{2}$ Institute for Physics of Microstructures, Russian Academy of Sciences, 603950 Nizhny Novgorod, GSP-105, Russia
}

(Received 17 July 2007; published 14 August 2007)

\begin{abstract}
The influence of an inhomogeneous magnetic field on the magnetoresistance of thin $\mathrm{Al} \mathrm{films,} \mathrm{used} \mathrm{in}$ different superconductor-ferromagnet hybrids, has been investigated. Two contrasting magnetic textures with out-of-plane magnetization are explored: namely, (i) a plain film in a multidomain state and (ii) an array of microsized dots. The stray fields of the ferromagnetic structures confine the superconducting condensate and, accordingly, modify the condition for the nucleation of superconductivity. By switching between different magnetic states of the ferromagnet, this confinement can be tuned at will, thereby reversibly changing the dependence of the critical temperature $T_{c}$ on an external magnetic field $H$. In particular, continuous evolution from a conventional linear $T_{c}(H)$ dependence with a single maximum to a reentrant superconducting phase boundary with multiple $T_{c}$ peaks has been demonstrated.
\end{abstract}

DOI: 10.1103/PhysRevB.76.060503

PACS number(s): 74.78.Fk, 74.25.Dw

The localization of a particle in a restricted volume is known to lead to a discrete energy spectrum due to the particle's wave nature. In some cases the trapping potential can be created artificially in a controlled way (e.g., in quantum dots and wells), and the geometry-dependent structure of the energy levels provides a convenient way to control the optical and transport properties of such objects. ${ }^{1}$ Remarkably, some basic concepts of standard quantum mechanics (including the confinement of the wave function) can also be applied to more complicated systems, like superconductors, in which a quantum condensate consisting of paired electrons develops. In this case, the energy of the lowest Landau level, $E_{L L L}(H)$, where $H$ is the external magnetic field, determines the critical temperature $T_{c}(H)$ at which superconductivity nucleates. ${ }^{2}$ Due to the strong dependence of $E_{L L L}(H)$ on the imposed confinement, $T_{c}(H)$ for superconducting microstructures and nanostructures differs significantly from that observed in bulk superconductors. ${ }^{3}$ Unfortunately, once this "geometrical" constraint is created, the trapping potential cannot be modified any longer.

However, the use of superconductor-ferromagnet (S-F) hybrids provides an appealing alternative to localize superconducting Cooper pairs. In such S-F hybrids the proximity effect $^{4}$ as well as the stray fields of the ferromagnet ${ }^{5}$ play an important role in changing the superconducting properties. A magnetic template which creates a nonuniform magnetic field distribution is able to localize the superconducting condensate (or normal electrons ${ }^{6}$ ). Such a modulated field profile can result in exotic shapes of the $T_{c}(H)$ phase boundary for S-F hybrids, revealing a simple shift of the $T_{c}$ maximum towards a certain magnetic field (so called field-induced-superconductivity 7,8 ) or a more complicated nonmonotonic $T_{c}(H)$ dependence with two maxima (reentrant superconductivity $\left.{ }^{9-11}\right)$, and are commonly explained in terms of magnetic field compensation effects. Indeed, for thin superconducting films, placed in a nonuniform magnetic field, superconductivity first nucleates near the $\left|B_{z}\right|$ minima, where $B_{z}$ is the out-of-plane component of the total magnetic field. ${ }^{9}$ The role of the nonuniform fields is simply to locally compensate an applied magnetic field, thereby enhancing superconductivity in the compensated area and consequently obtaining a maximum $T_{c}$ at some nonzero applied field. However, not only is the amplitude of the stray field, induced by the magnetic template, of importance ${ }^{11}$ (as follows from the idea of field compensation). According to the quantum size effect mentioned above, also the length scales of the area, where the compensation takes place, are crucial for the appearance of superconductivity. More precisely, localizing the superconducting order parameter (OP) in a wide region can result in a higher $T_{c}$ than a localized OP in a narrower region.

This work is aimed at demonstrating how tunable magnetic confinement of the superconducting order parameter can practically be realized. We show that this confinement is strongly dependent on the detailed structure of the underlying magnetic template. In addition, a reversible evolution of the $T_{c}(H)$ phase boundary can be obtained by changing the magnetic state of the template. These results bridge the gap between two apparently different subjects: domain-wall superconductivity and field-induced superconductivity.

In order to investigate the effects of the OP localization experimentally, two S-F hybrid samples with different ferromagnetic subsystems were investigated: a plain ferromagnetic film, containing bubble domains, and a square array of $1.52-\mu \mathrm{m}$-sized magnetic dots with a period of $2 \mu \mathrm{m}$. In both cases the ferromagnets consist of a $\operatorname{Pt}(2.5 \mathrm{~nm})$ buffer layer covered by a multilayer of $[\mathrm{Co}(0.4 \mathrm{~nm}) / \mathrm{Pt}(1.0 \mathrm{~nm})]_{n}$, where $n=15$ for the plain film and $n=10$ for the dots. The resulting magnetic structures show well-defined out-of-plane magnetization. ${ }^{12}$ Both templates are covered by a 5-nm-thick Si layer followed by a superconducting Al layer of $50 \mathrm{~nm}$ thickness. Since the Al film is insulated from the ferromagnetic substrate, the interaction between ferromagnet and superconductor is electromagnetic in origin with negligible proximity effects. Note that due to the low upper critical field of $\mathrm{Al}$, the nonuniform magnetic fields should have a stronger 

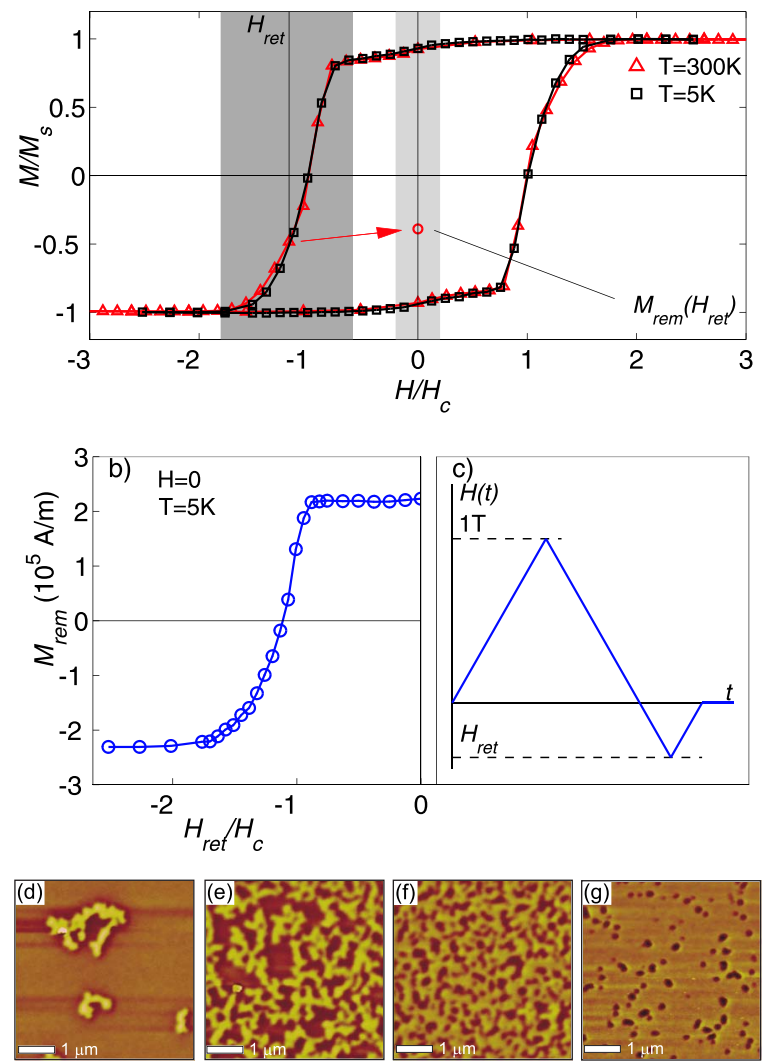

FIG. 1. (Color online) (a) Normalized magnetization loops $M(H)$ of the $\mathrm{Co} / \mathrm{Pt}$ plain film at $300 \mathrm{~K}(\triangle)$ and $5 \mathrm{~K}(\square)$. The magnetic field axis is normalized by the corresponding coercive fields. (b) Remanent magnetization $M_{\text {rem }}$, measured at $5 \mathrm{~K}$ and $H=0$ after saturation and subsequent application of a returning field $H_{\text {ret }}$ [this procedure is shown schematically in panel (c)]. (d)-(g) MFM pictures $\left(5 \times 5 \mu \mathrm{m}^{2}\right)$ obtained at $300 \mathrm{~K}$ for $H_{r e t} / H_{c}$ $=-0.92,-1.05,-1.31$, and -1.57 , respectively. The dark (bright) color represents domains with positive (negative) magnetization.

influence on the superconducting properties of an $\mathrm{Al}$ film in comparison with $\mathrm{Pb}$ or $\mathrm{Nb}$.

The magnetic properties of the plain $\mathrm{Co} / \mathrm{Pt}$ multilayer were investigated using a commercial Quantum Design superconducting quantum interference device (SQUID) magnetometer. Figure 1(a) shows the hysteresis loop at $5 \mathrm{~K}$ and $300 \mathrm{~K}$ after renormalization by their respective coercive fields $\mu_{0} H_{c}^{5 \mathrm{~K}}=397 \mathrm{mT}, \mu_{0} H_{c}^{300 \mathrm{~K}}=191 \mathrm{mT}$ and saturation magnetizations $M_{s}^{5 \mathrm{~K}}=2.25 \times 10^{5} \mathrm{~A} / \mathrm{m}, \quad M_{s}^{300 \mathrm{~K}}=2.45$ $\times 10^{5} \mathrm{~A} / \mathrm{m}$. Clearly the magnetization changes drastically for applied fields of the order of the coercive field $H_{c}$ [dark gray area in Fig. 1(a)]. This fact allows us to control the magnetization $M$ in zero externally applied field $H=0$. Indeed in Fig. 1(b) this remanent magnetization $M(H=0)$ is shown after saturating the film, applying a certain returning field $H_{r e t}$ and returning back to zero field [see Fig. 1(c)] for different $H_{r e t}$ values. Clearly any remanent magnetization between positive and negative saturation can be obtained by varying $H_{r e t}$. To investigate the microscopic domain distribution corresponding to these remanent magnetization states, magnetic force microscopy images were taken at room temperature. In Figs. 1(d)-1(g) MFM images are shown for a selected set of returning fields, giving details about the evolution from positive to negative magnetization. Having both hysteresis loops coincide nearly perfectly indicates that similar magnetization reversal processes occur at low temperatures. Accordingly, the domain distribution is expected to undergo a similar evolution at low temperatures as well. Thus by choosing the appropriate $H_{\text {ret }}$ value the desired domain distribution can be readily prepared. ${ }^{13}$ To control the magnetic state of the dots the same procedure can be applied since the diameter of the dots exceeds the typical size of the domains. Accordingly they are in a multidomain state ${ }^{7}$ and any intermediate remanent magnetization can be reached.

For a better understanding of the superconducting properties in the presence of an inhomogeneous magnetic profile, the $T_{c}(H)$ phase boundaries are calculated within GinzburgLandau theory and are compared with the experiment. As a simplest model we assume an infinitely thin superconducting film placed on top of a periodical one-dimensional (1D) domain structure [Figs. 2(a) and 2(b)]. We account for the controllable domain distribution by changing the ratio $\alpha$ of positively $L^{(+)}$and negatively $L^{(-)}$magnetized domains while keeping the period $L^{(+)}+L^{(-)}$constant. Although this relative weight $\alpha$ can be changed through a variation of the returning field $H_{r e t}$, we assume $\alpha$ to be constant when measuring the superconducting properties (for more details of the model see Ref. 14). The constancy of $\alpha$ is justified by the fact that the applied fields for measuring the superconductor are much smaller than the coercive field of the magnetic structures [see light and dark gray regions in Fig. 1(a)]. The calculated $T_{c}(H)$ phase boundaries are shown in Fig. 2(c) as a function of $\alpha$. The experimental phase boundaries are extracted from magnetoresistance measurements at different temperatures [see inset Fig. 2(d)], using a $80 \%$ criterium $^{15}$ of normal-state resistance, and are displayed in Fig. 2(d).

Both theoretical and experimental results show striking similarities which can be interpreted as follows. The phase boundary for a uniformly magnetized ferromagnetic film (curve $\alpha=0$ and curve I in Fig. 2) is linear since there are no domains inside the ferromagnet and, consequently, the effect of the magnetic stray field is negligible.

By applying a certain $H_{r e t}<0$, negative domains are introduced into the ferromagnetic film. For instance, for $H_{r e t} / H_{c}=-1.05$ [curve II in Fig. 2(d)] the net magnetization is reduced to about $33 \%$ of the saturation magnetization. The associated microscopic configuration is expected to be similar to the domain distribution shown in Fig. 1(e). By applying a negative external field, the stray field from the larger positive domains, can be compensated and superconductivity will nucleate locally above these domains, giving rise to a peak in the phase boundary located at negative fields. As a direct consequence of the increase of the ground energy of the "particle in a box," the maximal critical temperature decreases drastically as the width of the positive domain decreases (curves $\alpha=0.1, \alpha=0.2$ and curve II in Fig. 2).

A further increase of $\left|H_{\text {ret }}\right|$ leads to a more pronounced decrease of the positive domains, resulting in an even lower $T_{c}$. This peak is now located at even higher negative fields, since the absolute value of the $z$ component of the field increases with decreasing domain size [Fig. 2(b)]. Simultaneously, the growth of negatively magnetized domains re- 

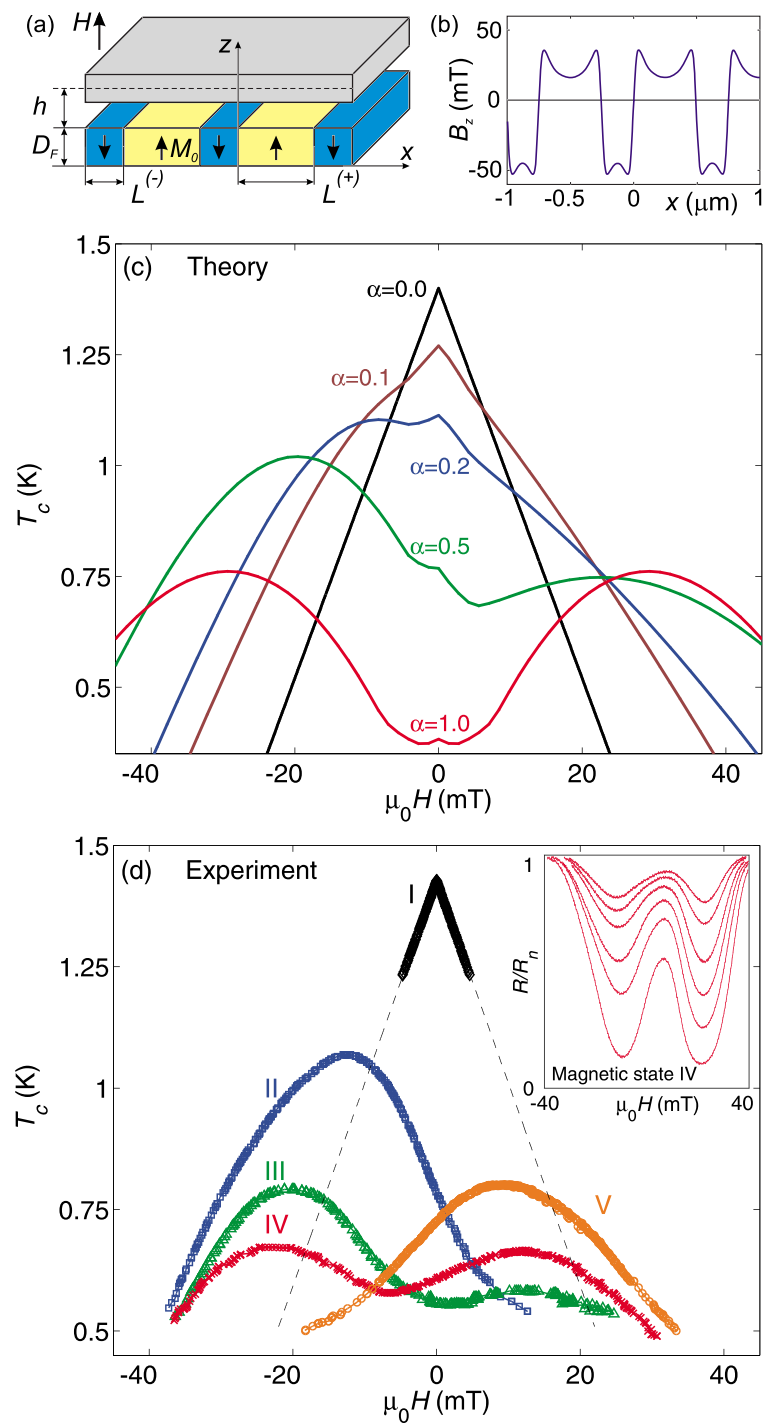

FIG. 2. (Color online) (a) Schematic presentation of an S-F bilayer with a 1D domain structure with different widths of positive $L^{(+)}$and negative $L^{(-)}$domains. (b) Profile of the $z$ component of the magnetic field, calculated for $L^{(-)} / L^{(+)}=0.5$ at a height of $h=30 \mathrm{~nm}$ using a saturation magnetization of $M_{s}=3.5 \times 10^{5} \mathrm{~A} / \mathrm{m}$ and a ferromagnetic film thickness $D_{F}=23 \mathrm{~nm}$. (c) Field dependence of the critical temperature $T_{c}$ of the considered S-F bilayer, calculated for several ratios of $\alpha=L^{(-)} / L^{(+)}$assuming the period $L^{(+)}+L^{(-)}=500 \mathrm{~nm}$ to be constant. The parameters of the Al film are chosen close to the experimentally determined values: critical temperature in zero field $T_{c 0}=1.4 \mathrm{~K}$ and coherence length $\xi(0)=100 \mathrm{~nm}$. (d) The experimental phase boundaries $T_{c}(H)$ for the bilayered sample in various magnetic states, obtained through different returning fields: $H_{r e t} / H_{c}=0$ (I), -1.05 (II), -1.11 (III), -1.15 $(\mathrm{IV})$, and $-1.21(\mathrm{~V})$. The inset shows magnetoresistance measurements for temperatures (top to bottom) $T=0.671 \mathrm{~K}, 0.642 \mathrm{~K}$, $0.610 \mathrm{~K}, 0.583 \mathrm{~K}, 0.551 \mathrm{~K}$, and $0.522 \mathrm{~K}$.

sults in a more favorable OP nucleation above negative domains and, accordingly, a second peak in the critical temperature at $H>0$ develops (curve $\alpha=0.5$ and curve III in Fig. 2 ). The relative amplitude of these peaks is determined by the specific details of the magnetic domain structure. For a returning field of $H_{r e t} / H_{c}=-1.15$ the remanent magnetization is close to zero, thus indicating the presence of an equal distribution of positive and negative domains. This domain structure gives rise to a nearly symmetric phase boundary (curve $\alpha=1$ and curve IV in Fig. 2). Similar phase boundaries with two maxima have already been observed in S-F hybrids, containing $\mathrm{Nb}$ and $\mathrm{Pb}$ films, and are attributed to domain wall superconductivity. ${ }^{10,11,16}$ For higher $H_{\text {ret }}$ values the first peak, located at negative fields, disappears, whereas the peak at positive fields shifts up in temperature and is displaced to a lower field (curve V in Fig. 2). This second peak will eventually evolve in a linear phase boundary when the ferromagnetic film is fully magnetized in the negative direction.

It is worth mentioning that the good agreement between the experimental results and our simplified 1D model indicates that the model used captures the essential physics behind the magnetic confinement effect very well. Yet a small discrepancy exists near $H=0$ where theory predicts a small peak which is not observed in the experiment. This peak corresponds to a wide OP distribution, spread over many periods of the magnetic field modulation. ${ }^{14} \mathrm{We}$ believe that this delocalized state is suppressed by the irregular shape of the domain distribution present in the real system.

We have applied the concept of tunable magnetic confinement also to another S-F hybrid system, consisting of a superconducting $\mathrm{Al}$ film covering an array of magnetic dots. The phase boundaries of such a structure in different mag-
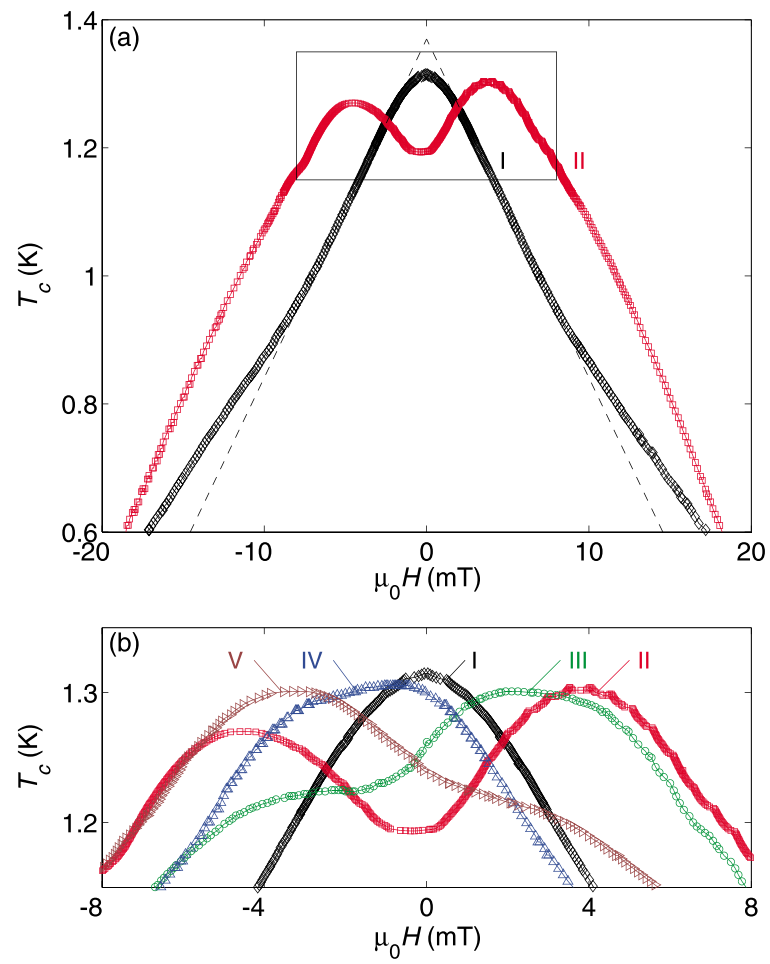

FIG. 3. (Color online) The phase boundaries $T_{c}(H)$ for an S-F hybrid, consisting of an $\mathrm{Al}$ film and an array of magnetic dots, in the demagnetized (I), the completely magnetized (II), and several intermediate magnetic states: $H_{r e t} / H_{c}=-0.99$ (III), -1.28 (IV), and $-1.54(\mathrm{~V})$, where $\mu_{0} H_{c}=227 \mathrm{mT}$. 
netic states are shown in Fig. 3. Due to the presence of the magnetic dots, three different areas, where the OP can be localized, are present: above the positive or negative domains inside the magnetic dot (similar to the bilayered system considered above) and between the dots, where the local magnetic field is roughly determined by the average magnetization of the dots. If the dots are magnetized positively, there is a negative field between the dots and vice versa. Note that there are no analogs of such regions with zero magnetization in the S-F bilayers.

In the demagnetized state (curve I in Fig. 3) the field in the region between the dots is approximately zero; accordingly, superconductivity starts to nucleate at this position at relatively low magnetic fields. As a result, an almost linear phase boundary centered at zero field is observed. At higher fields $\left(\mu_{0}|H| \geqslant 8 \mathrm{mT}\right)$ there is a clear deviation from the expected linear behavior. Such magnetic bias can be explained by the compensation of the magnetic field above the positive and negative domains inside the magnetic dot, similar to the bilayered sample presented above. Interestingly this phase boundary combines field compensation effects in each of the three regions.

By magnetizing the dots positively (i) the amplitude of the field in between the dots increases negatively and (ii) the typical size of the positive domains becomes larger than that for negative domains. As a result, the peak, associated with the OP localization in between the dots, shifts towards positive fields (field-induced superconductivity) and a second local $T_{c}$ maximum, corresponding to the appearance of superconductivity above the wider positive domains, appears, while the OP nucleation above narrower negative domains is suppressed (curves II and III in Fig. 3). For negatively magnetized dots the reversed effect occurs (curves IV and V in Fig. 3). It is important to note that the amplitude of the main $T_{c}$ peak remains almost constant when the magnetic state of dots is changed [compare curves I-V in Fig. 3(b)]. Indeed, since this peak corresponds to the nucleation of superconductivity in between the dots, the area of localization is almost independent of the dot's magnetic state.

Summarizing, we have studied tunable magnetic confinement of the superconducting OP in different S-F hybrids, which originates from nonuniform magnetic fields induced by a ferromagnetic template. By manipulating the domain structure in the ferromagnet through appropriate demagnetizing procedures, one can drastically change the position of the confined OP and, as a result, the shape of the phase boundary $T_{c}(H)$. In particular, restricting the area where optimal field compensation occurs is shown to induce a systematic reduction of the critical temperature of the superconducting transition. We have demonstrated that both domain-wall superconductivity and field-induced superconductivity are manifestations of the magnetic confinement effect in different magnetic structures.

This work was supported by the K.U. Leuven Research Fund GOA/2004/02 program, the Belgian IUAP, the Fund for Scientific Research, Flanders (F.W.O., Vlaanderen), the bilateral project BIL/05/25 between Flanders and Russia, by the Russian Foundation for Basic Research (A.Yu.A.), and by the F.W.O. (A.V.S.).
*Werner.Gillijns@fys.kuleuven.be

${ }^{1}$ E. E. Mendez and K. von Klitzing, Physics and Applications of Quantum Wells and Superlattices, NATO Advanced Studies Institute, Series B: Physics (Springer, Amsterdam, 1988), Vol. 170; P. Harrison, Quantum Wells, Wires and Dots: Theoretical and Computational Physics of Semiconductor Nanostructures, 2nd ed. (Wiley, Hoboken, NJ, 2005).

${ }^{2}$ M. Tinkham, Introduction to Superconductivity (McGraw-Hill, New York, 1996).

${ }^{3}$ V. V. Moshchalkov, L. Gielen, C. Strunk, R. Jonckheere, X. Qiu, C. Van Haesendonck, and Y. Bruynseraede, Nature (London) 373, 319 (1995); L. F. Chibotaru, A. Ceulemans, M. Morelle, G. Teniers, C. Carballeira, and V. V. Moshchalkov, J. Math. Phys. 46, 095108 (2005).

${ }^{4}$ A. I. Buzdin, Rev. Mod. Phys. 77, 935 (2005).

${ }^{5}$ I. F. Lyuksyutov and V. L. Pokrovsky, Adv. Phys. 54, 67 (2005).

${ }^{6}$ See, e.g., I. S. Ibrahim and F. M. Peeters, Phys. Rev. B 52, 17321 (1995); I. K. Marmorkos, A. Matulis, and F. M. Peeters, ibid. 53, 2677 (1996); J. Reijniers, F. M. Peeters, and A. Matulis, ibid. 59, 2817 (1999); S. L. Cheng and H. A. Fertig, ibid. 60, 13107 (1999).

${ }^{7}$ M. Lange, M. J. Van Bael, Y. Bruynseraede, and V. V. Moshchalkov, Phys. Rev. Lett. 90, 197006 (2003).

${ }^{8}$ W. Gillijns, A. V. Silhanek, and V. V. Moshchalkov, Phys. Rev. B 74, 220509(R) (2006).
${ }^{9}$ A. I. Buzdin and A. S. Mel'nikov, Phys. Rev. B 67, 020503(R) (2003); A. Yu. Aladyshkin, A. I. Buzdin, A. A. Fraerman, A. S. Melnikov, D. A. Ryzhov, and A. V. Sokolov, ibid. 68, 184508 (2003).

${ }^{10}$ Z. Yang, M. Lange, A. Volodin, R. Szymczak, and V. V. Moshchalkov, Nat. Mater. 3, 793 (2004).

${ }^{11}$ W. Gillijns, A. Yu. Aladyshkin, M. Lange, M. J. Van Bael, and V. V. Moshchalkov, Phys. Rev. Lett. 95, 227003 (2005).

${ }^{12}$ W. B. Zeper, F. J. A. M. Greidanus, P. F. Carcia, and C. R. Fincher, J. Appl. Phys. 65, 4971 (1989).

${ }^{13}$ M. Lange, M. J. Van Bael, V. V. Moshchalkov, and Y. Bruynseraede, Appl. Phys. Lett. 81, 322 (2002).

${ }^{14}$ A. Yu. Aladyshkin and V. V. Moshchalkov, Phys. Rev. B 74, 064503 (2006).

${ }^{15}$ This relatively high criterion allows us to separate the effects due to the confinement from the influence of different percolation paths. Indeed, by taking a high criterion, the onset of superconductivity is measured, which in turn is independent of the percolating path. As a result we assume any change in $T_{c}$ (determined with a high criterion) due to changes in the magnetic state of the magnetic layer mainly reflects localization and confinement of the order parameter.

${ }^{16}$ Z. Yang, J. Van de Vondel, W. Gillijns, W. Vinckx, V. V. Moshchalkov, and R. Szymczak, Appl. Phys. Lett. 88, 232505 (2006). 